\title{
The pleiotropic effects of fisetin and hesperetin on human acute promyelocytic leukemia cells are mediated through apoptosis, cell cycle arrest, and alterations in signaling networks
}

\author{
Aysun Adan ${ }^{1}$ Yusuf Baran ${ }^{1,2}$
}

Received: 6 April 2015 / Accepted: 19 May 2015 / Published online: 17 June 2015

(C) International Society of Oncology and BioMarkers (ISOBM) 2015

\begin{abstract}
Fisetin and hesperetin, flavonoids from various plants, have several pharmaceutical activities including antioxidative, anti-inflammatory, and anticancer effects. Howev$\mathrm{er}$, studies elucidating the role and the mechanism(s) of action of fisetin and hesperetin in acute promyelocytic leukemia are absent. In this study, we investigated the mechanism of the antiproliferative and apoptotic actions exerted by fisetin and hesperetin on human HL60 acute promyelocytic leukemia cells. The viability of HL60 cells was evaluated using the MTT assay, apoptosis by annexin V/propidium iodide (PI) staining and cell cycle distribution using flow cytometry, and changes in caspase- 3 enzyme activity and mitochondrial transmembrane potential. Moreover, we performed wholegenome microarray gene expression analysis to reveal genes affected by fisetin and hesperetin that can be important for developing of future targeted therapy. Based on data obtained from microarray analysis, we also described biological networks modulated after fisetin and hesperetin treatment by KEGG and IPA analysis. Fisetin and hesperetin treatment showed a concentration- and time-dependent inhibition of proliferation and induced G2/M arrest for both agents and G0/G1 arrest for hesperetin at only the highest concentrations. There was a disruption of mitochondrial membrane potential together with increased caspase-3 activity. Furthermore, fisetin- and hesperetin-triggered apoptosis was confirmed by annexin V/PI analysis. The microarray gene profiling analysis
\end{abstract}

Yusuf Baran

yusufbaran@iyte.edu.tr

1 Department of Molecular Biology and Genetics, İzmir Institute of Technology, 35430 Urla, İzmir, Turkey

2 Faculty of Life and Natural Sciences, Abdullah Gul University, 38080 Kayseri, Turkey revealed some important biological pathways including mitogen-activated protein kinases (MAPK) and inhibitor of DNA binding (ID) signaling pathways altered by fisetin and hesperetin treatment as well as gave a list of genes modulated $\geq 2$-fold involved in cell proliferation, cell division, and apoptosis. Altogether, data suggested that fisetin and hesperetin have anticancer properties and deserve further investigation.

Keywords Fisetin $\cdot$ Hesperetin $\cdot$ Apoptosis $\cdot$ Acute promyelocytic leukemia $\cdot$ Gene profiling

\section{Introduction}

Acute promyelocytic leukemia (APL), a distinct variant of acute myeloid leukemia (AML), is different from other types of AML with its molecular biology and clinical outcomes. APL is characterized by abnormal accumulation of immature granulocytes (promyelocytes) in the bone marrow and blood stream [1]. In the majority of APL cases, translocation, $\mathrm{t}(15 ; 17)$, between the retinoic acid receptor-alpha (RAR $\alpha)$ gene on chromosome 17 and the promyelocytic leukemia (PML) gene on chromosome 15 is very common, which is responsible for the pathogenesis of APL [2]. Even though several treatment strategies are available for APL such as chemotherapy including all-trans retinoic acid (ATRA) and arsenic trioxide (ATO) and stem cell transplantation, they remain incurable due to their heterogenic nature, differences in their biological characteristics, drug resistance, and high recurrence rate and harmful side effects [3]. Thus, efforts to develop new treatments for APL are necessary.

Recently, dietary phytochemicals targeting cellular pathways in tumor cells have comprised great interest to develop novel apoptosis-based therapies [4]. Of these phytochemicals, flavonoids widely distributed in plants have received 
increasing attention for their potential chemopreventive role, because they have been known to impair cancer cell growth by modulating various signaling pathways involved in different stages of cancer development as well as inducing apoptosis [5].

Fisetin (3,3', 4',7-tetrahydroxyflavone), a bioactive flavanol molecule, is found in various fruits and vegetables, such as strawberry, apple, persimmon, grape, onion, and cucumber [6]. Fisetin has a broad range of biological activities including antioxidant, anti-inflammatory, and anticancer effects, and its chemotherapeutic potential has been studied on various in vitro and in vivo cancer models [7]. Despite that fisetin has been shown to induce apoptosis in a variety of cell lines, fisetin-mediated antiproliferative and apoptotic effects were specific for cancer cells based on differential modulation of cellular signaling pathways. The mechanisms behind fisetin's actions include induction of cell cycle arrest by altering cell cycle regulators [8], inhibition of signaling pathways such as NF-KB and PI3K/Akt pathways [9, 10], prevention of cell invasion and metastasis [11], and modulation of apoptotic and anti-apoptotic molecules [12]. These effects of fisetin have been evaluated on various cancer types such as colon, prostate, pancreas, and lung cancers; however, there is no study investigating the underlying mechanism(s) of fisetin in APL.

Citrus species have attracted significant attention for their flavonoid content which is thought to be promising in cancer therapy. Hesperidin and hesperetin are the most important examples of these flavonones found in citrus fruits such as lemon and orange with a wide range of pharmacological properties including antioxidant, anti-inflammatory, and anticancer effects [13]. Hesperidin could be considered as a pro-drug, since it is metabolized to hesperetin before absorption [14]. Promising results derived from a noticeable body of evidence displayed that hesperetin could mediate its anticancer effects in several cancer types by affecting several cellular targets. Some of these mechanisms include modulation of apoptotic proteins [15], increased activity of several caspases like caspase-3 and caspase-9 [16], and arrest of cell cycle progression [17]. On the other hand, the mechanism(s) responsible for the biological effects of hesperetin in APL cells remain unidentified yet.

The present study was undertaken to determine the molecular mechanism of fisetin- and hesperetin-induced cell death in HL60 APL cells by investigating their effects on cell proliferation, apoptosis, caspase-3 enzyme activity, loss of mitochondrial membrane potential, and cell cycle distribution. Moreover, whole-genome microarray analysis was performed to establish affected genes and genetic networks in response to fisetin and hesperetin. This is the first report that demonstrates the therapeutic potential of these flavanoids in APL cells via inducing apoptosis and cell cycle arrest and modulating multiple cellular targets.

\section{Materials and methods}

\section{Chemicals}

Fisetin (purity $\geq 98 \%$ ) and hesperetin (purity $\geq 95 \%$ ) were obtained from Santa Cruz Biotechnology, Inc. (Heidelberg, Germany). Ten-millimolar stock solutions of these agents were prepared in DMSO and stored at $-20^{\circ} \mathrm{C}$. The final concentration of DMSO did not exceed more than $0.1 \%$ in culture. Penicillin-streptomycin, RPMI 1640, and fetal bovine serum were obtained from Invitrogen (Paisley, UK). MTT reagent was purchased from Sigma (St. Louis, MO).

\section{Cell culture}

Human HL60 APL cells were kindly provided by Dr. Ali Ugur Ural from the Hematology Department of Gulhane Medical School, cultured in RPMI 1640 growth medium supplemented with $10 \%$ fetal bovine serum and $1 \%$ penicillinstreptomycin at $37^{\circ} \mathrm{C}$ in $5 \% \mathrm{CO}_{2}$.

\section{MTT assay}

Antiproliferative effects of fisetin and hesperetin on HL60 cells were assessed by the MTT cell proliferation assay, which depends on reduction of MTT dye to its insoluble formazan by NAD(P)H-dependent cellular oxidoreductase enzymes, giving a purple color in living cells. Briefly, $1 \times 10^{4}$ cells grown in 96-well plates were exposed to increasing concentrations of fisetin and hesperetin $(1-200 \mu \mathrm{M})$ for 48 and $72 \mathrm{~h}$. These cells were then incubated with $20 \mu \mathrm{l}$ MTT for $3 \mathrm{~h}$, and the resulting formazan crystals were dissolved in $100 \mu \mathrm{LMSO}$. The absorbance values were read at 570-nm wavelength with an ELISA reader (Thermo Electron Corporation Multiskan Spectrum, Finland). Finally, the $\mathrm{IC}_{50}$ value (drug concentration which inhibits cell growth by $50 \%$ ) of these agents was calculated from cell proliferation plots.

\section{Assessment of apoptosis in human HL60 cells by annexin V-FITC/PI double staining}

To examine apoptotic effects of fisetin and hesperetin on APL cells, the movement of phosphatidylserine (PS) from the inner leaflet of the membrane to the outer leaflet can be easily detected by using a fluorescent conjugate of annexin V (Annexin V-FITC Apoptosis Detection Kit, BioVision Research Products, USA). A total of $5 \times 10^{5}$ cells/well seeded into six-well plates were treated with increasing concentrations of agents for $72 \mathrm{~h}$. Then, the collected cells were washed twice with cold PBS, suspended with $200 \mu \mathrm{l}$ of $1 \times$ binding buffer, and stained with $2 \mu \mathrm{l}$ of FITC Annexin V and $2 \mu \mathrm{l}$ of propidium iodide (PI) for $15 \mathrm{~min}$ at room temperature in the dark. Afterwards, 
samples were analyzed by flow cytometry (BD Facscanto Flowcytometry, Belgium) within $1 \mathrm{~h}$.

\section{Detection of the changes in mitochondrial membrane potential}

The effects of fisetin and hesperetin on mitochondrial membrane potential (MMP) of HL60 cells were examined by JC-1 Mitochondrial Membrane Potential Detection Kit (Cayman Chemicals, USA), which uses a unique cationic dye, JC-1. JC-1 has the ability to enter into mitochondria and change its color from green to red reversibly. In healthy cells with high MMP, JC-1 spontaneously forms complexes in mitochondria and gives intense red fluorescence. On the other hand, in apoptotic cells with low MMP, JC-1 remains in the monomeric form and gives green fluorescence. Briefly, $5 \times 10^{5}$ cells/well seeded into six-well plates were treated with agents for $72 \mathrm{~h}$ and then collected by centrifugation at $180 \mathrm{~g}$ for $10 \mathrm{~min}$. The pellets were homogenized with $300 \mu \mathrm{l}$ medium containing $30 \mu \mathrm{l}$ of JC- 1 dye and incubated at $37{ }^{\circ} \mathrm{C}$ in $5 \% \mathrm{CO}_{2}$ for $30 \mathrm{~min}$. Then, the cells were collected by centrifugation at $400 \mathrm{~g}$ for $5 \mathrm{~min}$ and homogenized with $200 \mu \mathrm{l}$ assay buffer. Afterwards, $100 \mu \mathrm{l}$ from each sample was added to a 96-well plate as triplicates. In healthy cells, the aggregate red form has absorption/emission maxima of 560/595 nm, whereas in apoptotic cells, the monomeric green form has absorption/ emission maxima of $485 / 535 \mathrm{~nm}$, which were read by a fluorescence ELISA reader (Thermo Varioskan Spectrum, Finland). The ratio of fluorescent intensity of JC-1 monomers (green) to fluorescent intensity of JC-1 aggregates (red) was calculated for each concentration as well as for the untreated control sample.

\section{Measurement of caspase- 3 activity}

Changes in caspase- 3 activity of the cells were examined by caspase-3 colorimetric assay kit (BioVision Research Products, USA), which is based on spectrophotometric detection of the chromophore $p$-nitroanilide ( $p \mathrm{NA}$ ) released from the labeled substrate DEVD- $p$ NA after cleavage by caspases. Briefly, the cells $\left(5 \times 10^{5}\right.$ cells/well seeded into six-well plates $)$ induced to undergo apoptosis by fisetin and hesperetin were collected by centrifugation at $180 \mathrm{~g}$ for $10 \mathrm{~min}$, which were then lysed by $50 \mu \mathrm{l}$ of chilled cell lysis buffer on ice for $10 \mathrm{~min}$. The reaction cocktail including $50 \mu \mathrm{l}$ of $2 \times$ reaction buffer (containing $10 \mathrm{mM}$ DTT), $50 \mu \mathrm{l}$ of sample, and $5 \mu \mathrm{l}$ of DEVD-pNA substrate was prepared and then added to 96well plates, which was incubated for $2 \mathrm{~h}$ at $37^{\circ} \mathrm{C}$. Finally, the plate was read under 405 -nm wavelengths by an ELISA reader. Total protein concentrations determined by the Bradford method were used to normalize the absorbance values.

\section{Cell cycle analysis by flow cytometry}

dsDNA content and distribution of cell cycle phases are determined by flow cytometry. Briefly, $5 \times 10^{5}$ cells/well were treated with increasing concentrations of fisetin and hesperetin for $72 \mathrm{~h}$. Then, the cells collected by centrifugation at $260 \mathrm{~g}$ for $10 \mathrm{~min}$ were homogenized with $1 \mathrm{ml}$ cold PBS and fixed with $4 \mathrm{ml}$ of cold ethanol overnight at $-20{ }^{\circ} \mathrm{C}$ for the analysis. The cells were centrifuged at $260 \mathrm{~g}$ for $10 \mathrm{~min}$, and pellets were homogenized with $1 \mathrm{ml}$ cold PBS and centrifugation was repeated. Afterwards, cell pellets were homogenized with $1 \mathrm{ml}$ PBS containing $0.1 \%$ Triton X-100, and then $100 \mu \mathrm{l}$ RNase A $(200 \mu \mathrm{g} / \mathrm{ml})$ was added and incubated at $37{ }^{\circ} \mathrm{C}$ for $30 \mathrm{~min}$. One hundred microliters of PI $(1 \mathrm{mg} / \mathrm{ml})$ was added to the cells, which were incubated at room temperature for $15 \mathrm{~min}$, and then analyzed by flow cytometry.

\section{Microarray analysis}

Total RNA was isolated from HL60 cells treated with fisetin $(20$ and $50 \mu \mathrm{M})$ and hesperetin $(100$ and $150 \mu \mathrm{M})$ for $72 \mathrm{~h}$ using Nucleospin Total RNA isolation kit (Machery-Nagel, USA) according to the manufacturer's instructions. Only the RNA samples with an A260/A280 ratio between 1.9 and 2.1 were considered for further use. Expression profiling is accomplished using the Illumina Human HT-12v4 beadchip microarrays (containing 47,000 transcripts: $\sim 30,000$ genes) (Illumina, Inc., San Diego, CA). Initially, $500 \mathrm{ng}$ of total RNA converted to cDNA, followed by in vitro transcription to generate biotin-labeled cRNA using the Illumina Total Prep RNA Amplification Kit (Ambion, USA) based on the manufacturer's instructions for hybridization analysis. Then, $1.5 \mu \mathrm{g}$ of labeled cRNA was hybridized to each array according to the Illumina whole-genome gene expression direct hybridization assay protocol. Then, arrays were imaged using the Illumina BeadArray Reader to measure fluorescence intensity at each probe. The images were processed and converted into signal intensities using the Illumina GenomeStudio software (Illumina, Inc.). Gene ontology and pathway analyses were performed to consider the biological meaning of differential expression of genes between the treated and untreated samples by using the Kyoto Encyclopedia of Genes and Genomes (KEGG) and Ingenuity Pathway Analysis (IPA; Ingenuity Systems, Redwood City, CA).

\section{Statistical analysis}

Statistical significance was determined using one-way analysis of variance (ANOVA) for MTT analyses and two-way ANOVA for annexin V, MMP, caspase-3 activity, and cell cycle analyses. $P<0.05$ was considered to be significant. All 
experiments were repeated in triplicate. Hierarchical cluster analysis (Illumina Genome Studio) applied to data sets to evaluate the "proximity" between genes and hierarchical clusters was constructed with the statistically significant $(P<0.05)$ genes. Genes were considered differentially expressed when logarithmic gene expression ratios in three independent hybridizations were more than 1.5 or less than 0.66 and when the $P$ values were less than 0.05 . For each comparison, we obtained the list of differentially expressed genes constrained by $P$ value $<0.05$ and at least 2.0 fold change.

\section{Results}

\section{The growth inhibitory effects of fisetin and hesperetin on HL60 cells}

To characterize the effects of fisetin and hesperetin on HL60 cells' growth, the viability of the cells treated with agents $(1-200 \mu \mathrm{M})$ for 48 and $72 \mathrm{~h}$ was measured using an MTT assay. Results showed that fisetin was effective in inhibiting the growth in a dose- and time-dependent manner, whereas hesperetin showed significant effect on the proliferation of the cells in a dose-dependent way especially after $72 \mathrm{~h}$ of incubation. For $48 \mathrm{~h}$ of hesperetin treatment, growth inhibition was not significant up to $100 \mu \mathrm{M}$. $\mathrm{IC}_{50}$ (inhibition of cell viability by $50 \%$ at a particular concentration) values at 48 and $72 \mathrm{~h}$ were calculated from cell proliferation plots and were found to be 82 and $45 \mu \mathrm{M}$ for fisetin (Fig. 1a) and 190 and $142 \mu \mathrm{M}$ for hesperetin, respectively (Fig. 1b).

\section{Fisetin and hesperetin trigger apoptosis in HL60 cells}

HL60 cells were incubated with increasing concentrations of fisetin and hesperetin for $72 \mathrm{~h}$, and fisetin- and hesperetin-induced changes in apoptosis (late apoptotic plus early apoptotic population) were detected by flow cytometry using annexin V-FITC and propidium iodide (PI) staining. Figure 2 shows the fold changes in the percentage of apoptotic HL60 cells as compared to untreated controls. Fisetin treatment resulted in 1.6-, 6.2-, and 11.6-fold increases in apoptosis in response to 20,50 , and $100 \mu \mathrm{M}$ fisetin, respectively (Fig. 2a). On the other hand, there were 1.2-, 2.1-, and 3.3-fold increases in response to 50, 100 , and $200 \mu \mathrm{M}$ hesperetin, respectively (Fig. 2c). Figure $2 b, d$ indicates flow data representing the percentage of the cells in each flow quadrant. Fisetin was found to be the most effective flavonoid for the induction of apoptosis in HL60 cells based on the detection of PS on the outer membrane.
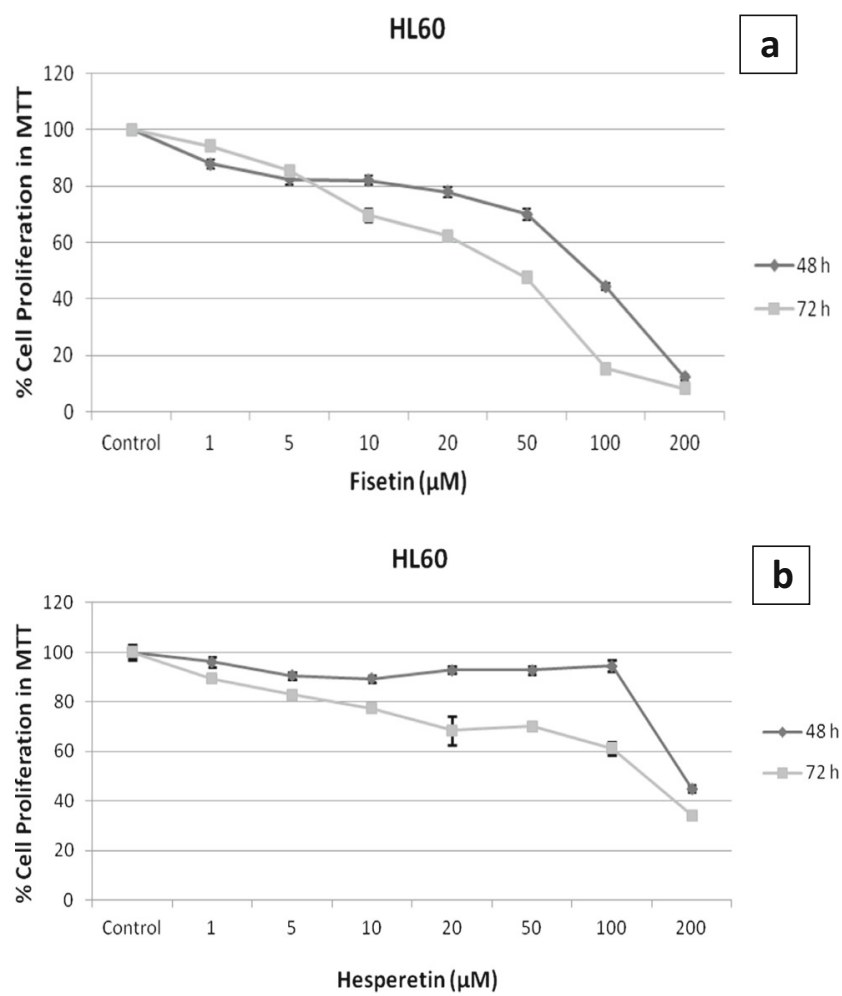

Fig. 1 Antiproliferative effects of fisetin (a) and hesperetin (b) on HL60 cells by the MTT assay. The $\mathrm{IC}_{50}$ values were calculated from cell proliferation plots. The results, the means of three independent experiments, are presented as mean $\pm \mathrm{SEM}$; when not seen, they are smaller than the thickness of the lines on the graphs. Statistical significance was determined using one-way analysis of variance and $P<0.05$ was considered to be significant

Fisetin and hesperetin induce loss of mitochondrial membrane potential in HL60 cells in a dose-dependent manner

Loss of mitochondrial membrane potential is an important sign of apoptosis since it has been linked to initiation and activation of apoptotic cascades [18]. Therefore, it was determined whether these flavonoids cause the loss of MMP in APL cells exposed to increasing concentrations of them by using a JC-1 dye-based method. Treatment of HL60 cells with 20,50 , and $100 \mu \mathrm{M}$ fisetin caused 1.01-, 1.15-, and 1.37-fold increases in loss of MMP, respectively (Fig. 3a). Figure 3b shows that hesperetin treatment resulted in 1.9-, 2.4-, and 5.4-fold increases in response to 50,100 , and $200 \mu \mathrm{M}$, respectively.

\section{Fisetin and hesperetin increase caspase-3 activity in HL60 cells}

To determine whether apoptosis induced by fisetin and hesperetin was associated with activation of caspase-3, a hallmark of apoptosis, the cells were treated with increasing concentrations of these agents for $72 \mathrm{~h}$ and the changes in 


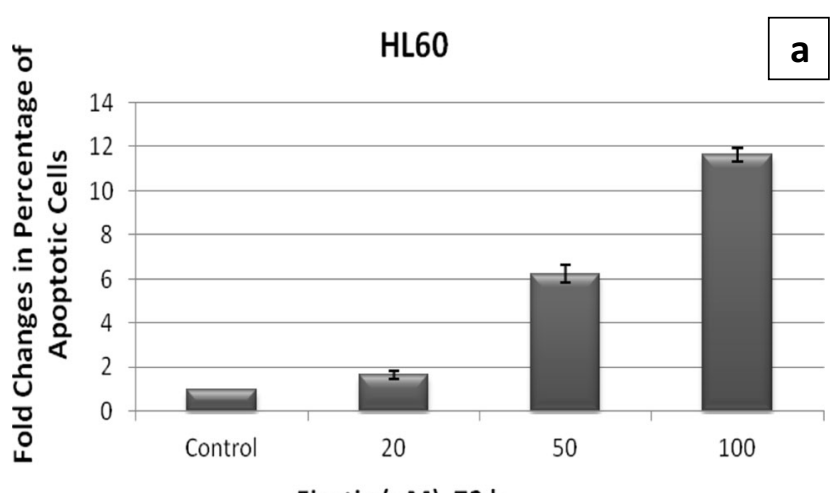

Fisetin ( $\mu \mathrm{M}), 72 \mathrm{~h}$

b

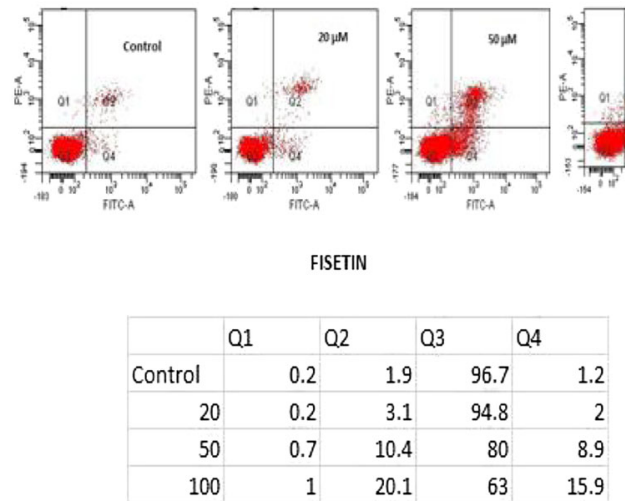

Fig. 2 Apoptotic effects of fisetin (a, b) and hesperetin (c, d) on HL60 cells by FACS analysis via annexin V-FITC/PI staining. Cells in the lower right quadrant indicate annexin-positive/PI-negative (Q4), early apoptotic cells. Cells in the upper right quadrant indicate annexinpositive/PI-positive, late apoptotic $(Q 2)(\mathbf{b}, \mathbf{d})$. The results, the means of

caspase-3 enzyme activity were detected through the cleavage of the labeled substrate DEVD- $p$ NA. As indicated in Fig. 4a, there were 1.05-, 2.0-, and 2.2-fold increases in caspase-3 activity of fisetin-treated HL60 cells in response to 20,50, and $100 \mu \mathrm{M}$, respectively, as compared to untreated controls. On the other hand, treatment of HL60 cells with 50, 100, and $200 \mu \mathrm{M}$ hesperetin caused 1.08-, 1.1-, and 2.8-fold increases in caspase-3 enzyme activity, respectively (Fig. $4 \mathrm{~b}$ ).

\section{Fisetin arrested cell cycle at the $\mathbf{G} 2 / \mathbf{M}$ phase while hesperetin at both G0/G1 and G2/M in HL60 cells}

In order to determine the possible mechanism of antiproliferative activity of the agents, cell cycle profiles of APL cells in response to $72 \mathrm{~h}$ of exposure to fisetin and hesperetin were examined by flow cytometry in the presence of DNase-free RNase and PI dye. Treatment of HL60 cells with fisetin resulted in dose-dependent increases in the percentage of cells in the $\mathrm{G} 2 / \mathrm{M}$ phase, which was accompanied by a reduction in the percentage of cells in the G0/G1 phase. The G2/M phase cell cycle distribution was $0.89,8$, and $29.5 \%$ at $20-, 50-$, and $100-\mu \mathrm{M}$ concentrations of fisetin, respectively (control value
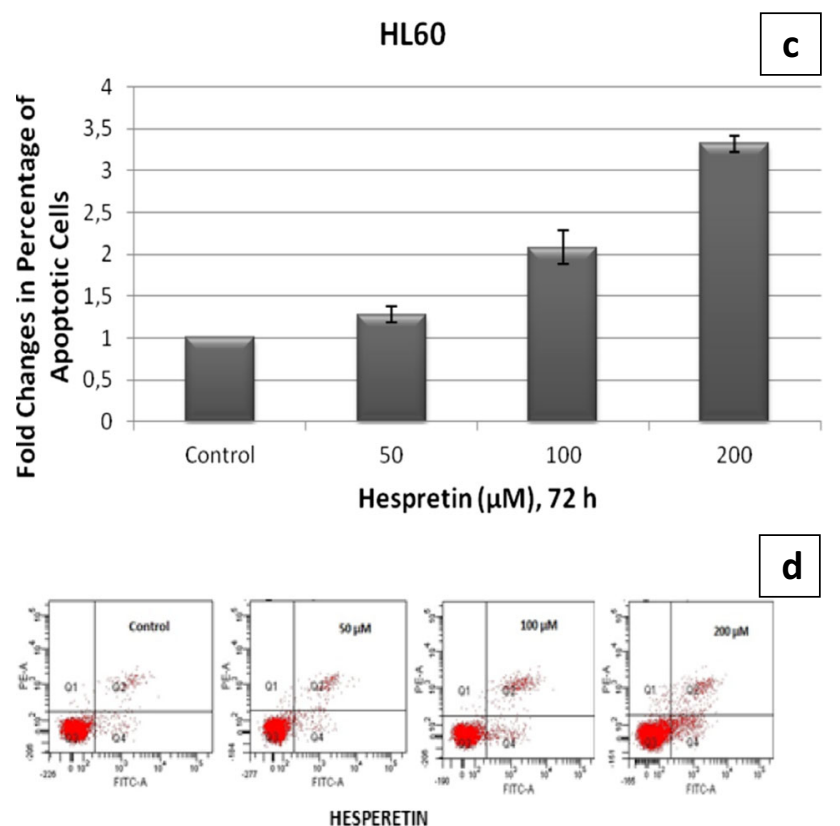

\begin{tabular}{|r|r|r|r|r|r|}
\hline & Q1 & & Q3 & Q4 \\
\hline Control & 0,1 & 1,3 & 97,4 & 1,2 \\
\hline 50 & 0,2 & 1,8 & 96,6 & 1,4 \\
\hline 100 & 0,1 & 3,2 & 94,7 & 2 \\
\hline 200 & 0,5 & 3 & 91,1 & 5,3 \\
\hline
\end{tabular}

three independent experiments, are presented as mean \pm SEM; when not seen, they are smaller than the thickness of the lines on the graphs. Statistical significance was determined using two-way analysis of variance and $P<0.05$ was considered to be significant

$0.57 \%$ ) (Fig. 5a). On the other hand, hesperetin treatment was found to result in a dose-dependent increase of cell population in the $\mathrm{G} 2 / \mathrm{M}$ phase. The $\mathrm{G} 2 / \mathrm{M}$ phase cell cycle distribution was $1.64,3.65$, and $13 \%$ at 50-, 100-, and $200-\mu \mathrm{M}$ concentrations of hesperetin, respectively (control value $0.46 \%$ ). Moreover, the percentage of the cells in the G0/G1 phase was found to increase especially at $100(34.8 \%)$ and 200 (44.3\%) $\mu \mathrm{M}$ hesperetin as compared to control (31.13\%) while there was a dose-dependent decrease in the $\mathrm{S}$ phase population (Fig. 5b).

\section{Microarray analysis detects differentially expressed genes affected by fisetin and hesperetin in HL60 cells}

We examined the changes in expression levels of the genes following treatment of HL60 cells with 20 and $50 \mu \mathrm{M}$ fisetin and 100 and $150 \mu \mathrm{M}$ hesperetin. Isolated total RNA was amplified and converted to biotin-labeled cRNA, which was hybridized to a microarray system containing approximately 30 , 000 genes. Hierarchical clustering of gene expression in untreated (control), fisetin-treated, and hesperetin-treated HL60 cells is shown in Fig. 6. 
a

HL60
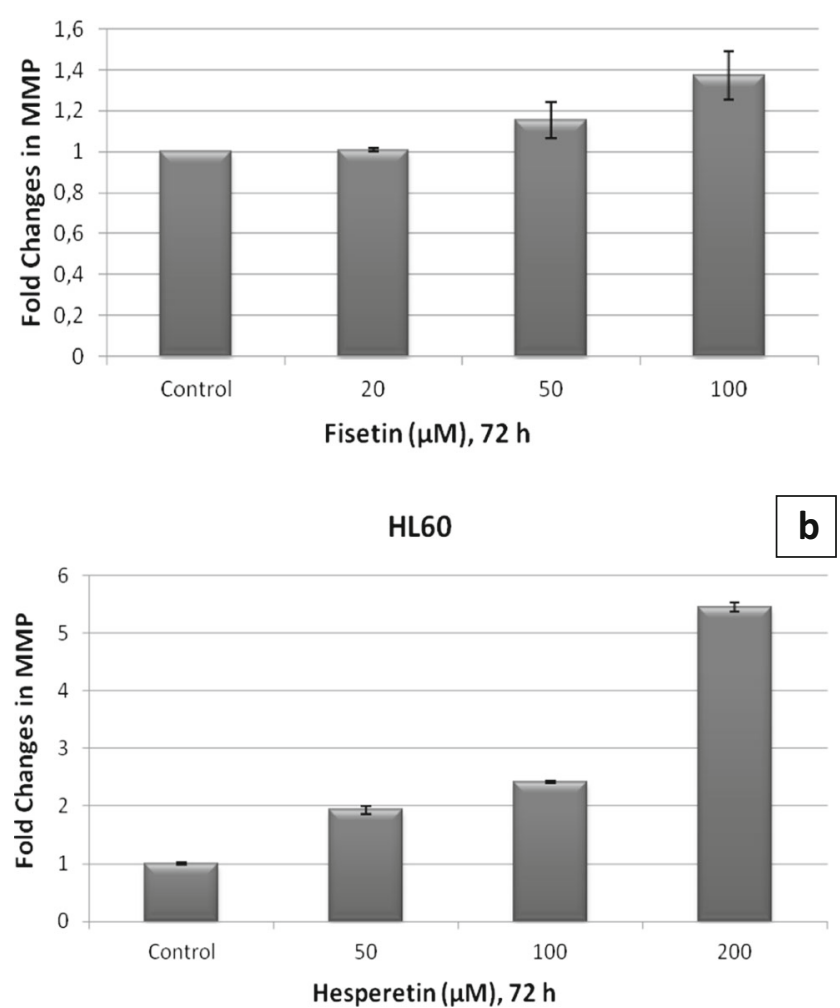

Fig. 3 Changes in MMP of HL60 cells treated with increasing concentrations of fisetin (a) and hesperetin (b). The results, the means of three independent experiments, are presented as mean \pm SEM; when not seen, they are smaller than the thickness of the lines on the graphs. Statistical significance was determined using two-way analysis of variance and $P<0.05$ was considered to be significant

A total of 54 and 1608 genes were significantly regulated $(P<0.05)$ in 20 - and $50-\mu \mathrm{M}$-fisetin-treated HL60 cells, respectively. The number of genes upregulated was 30 whereas that of genes downregulated was 24 in $20-\mu \mathrm{M}$-fisetin-treated HL60 cells. In 50- $\mu \mathrm{M}$-fisetin-treated HL60 cells, 527 genes were upregulated and 1081 genes were downregulated. Fold change analysis displayed that TXNIP (thioredoxininteracting protein), TFPI (tissue factor pathway inhibitor), miRNA1974, ID1 and ID3 (inhibitor of DNA binding 1 and 3), HSPA1B (heat shock protein 1B), and IDH1 (isocitrate dehydrogenase 1, NADP+) were altered genes in both 20 and $50-\mu \mathrm{M}$-fisetin-treated HL60 cells (Table 1). It was clear that MAP3K1 (mitogen-activated protein kinase kinase kinase 1), caspase-4, LASS6 (longevity assurance gene 6), and CBLB (E3 ubiquitin-protein ligase CBL-B) were the examples of upregulated genes, while LONP1 (Lon protease 1), STAT5A and STAT3 (signal transducer and activator of transcription 5A and 3), and JAK1 (Janus kinase 1) were some of the downregulated genes in 50- $\mu \mathrm{M}$-fisetin-treated HL60 cells (Table 1).

On the other hand, a total of 130 and 691 genes were significantly altered $(P<0.05)$ in $100-$ and $150-\mu \mathrm{M}$ -
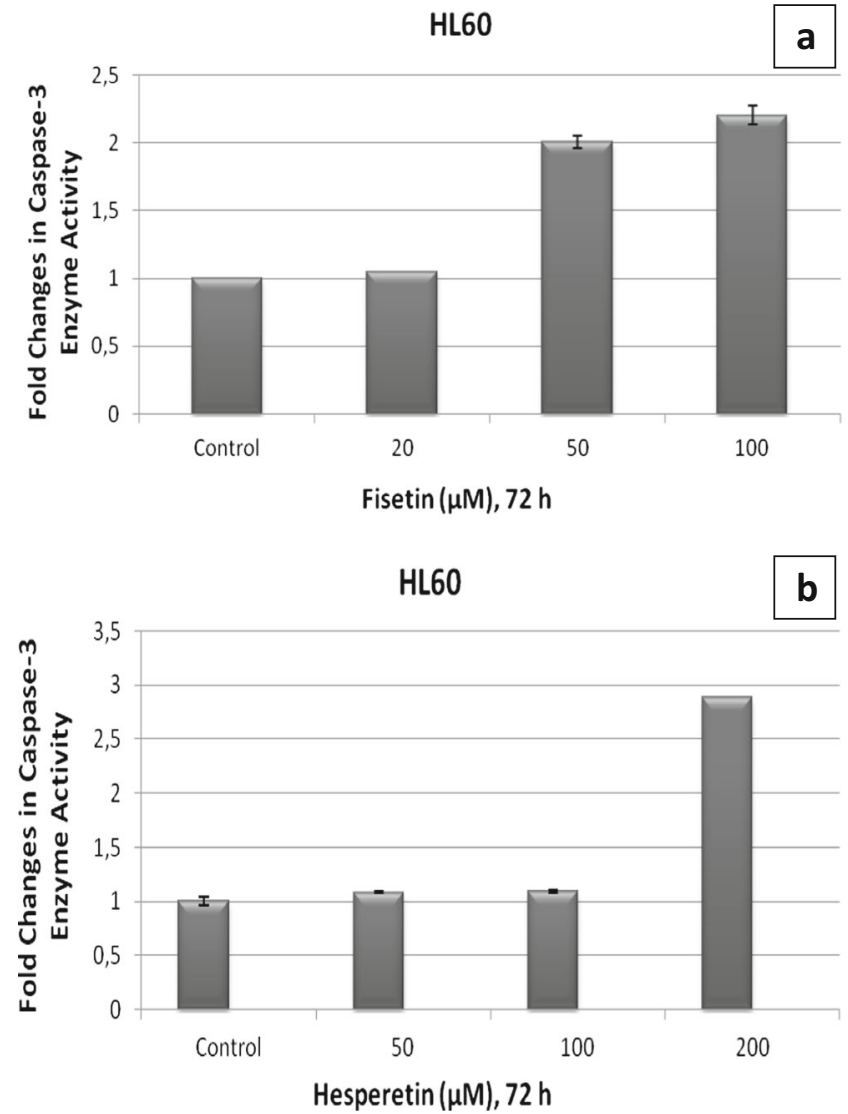

Fig. 4 Changes in caspase-3 enzyme activity in response to fisetin and hesperetin in HL60 cells. The results, the means of three independent experiments, are presented as mean $\pm \mathrm{SEM}$; when not seen, they are smaller than the thickness of the lines on the graphs. Statistical significance was determined using two-way analysis of variance and $P<0.05$ was considered to be significant

hesperetin-treated HL60 cells, respectively. Treatment with $100 \mu \mathrm{M}$ hesperetin resulted in the upregulation of 36 genes and downregulation of 94 genes. The number of genes upregulated was 288, whereas that of genes downregulated was 403 in 150- $\mu \mathrm{M}$-hesperetin-treated HL60 cells. SASH1 (SAM and SH3 domain-containing protein 1), MT1F (metallothionein 1F), and SPRR2D (small proline-rich protein 2D) were representative common upregulated genes, while TUBB1 (tubulin beta-1 chain), ID3, ID1, NMU (neuromedin U), FGFR3 (fibroblast growth factor receptor 3), and S100P (calciumbinding protein $\mathrm{P}$ ) were common downregulated genes in both 100- and $150-\mu \mathrm{M}$-hesperetin-treated HL60 cells (Table 2) based on fold change analysis. Furthermore, $150 \mu \mathrm{M}$ hesperetin induced more genes that were either upregulated or downregulated as compared to $100 \mu \mathrm{M}$ hesperetin (Table 2). TXNIP, MT1A (metallothionein 1A), MAP3K1, and SPRR2F (small proline-rich protein $2 \mathrm{~F}$ ) were some of the upregulated genes, while RPS25 (ribosomal protein S25), C-MYC, and tubulin family members (TUBA1C and TUBB2C) were examples of downregulated genes (Table 2). 

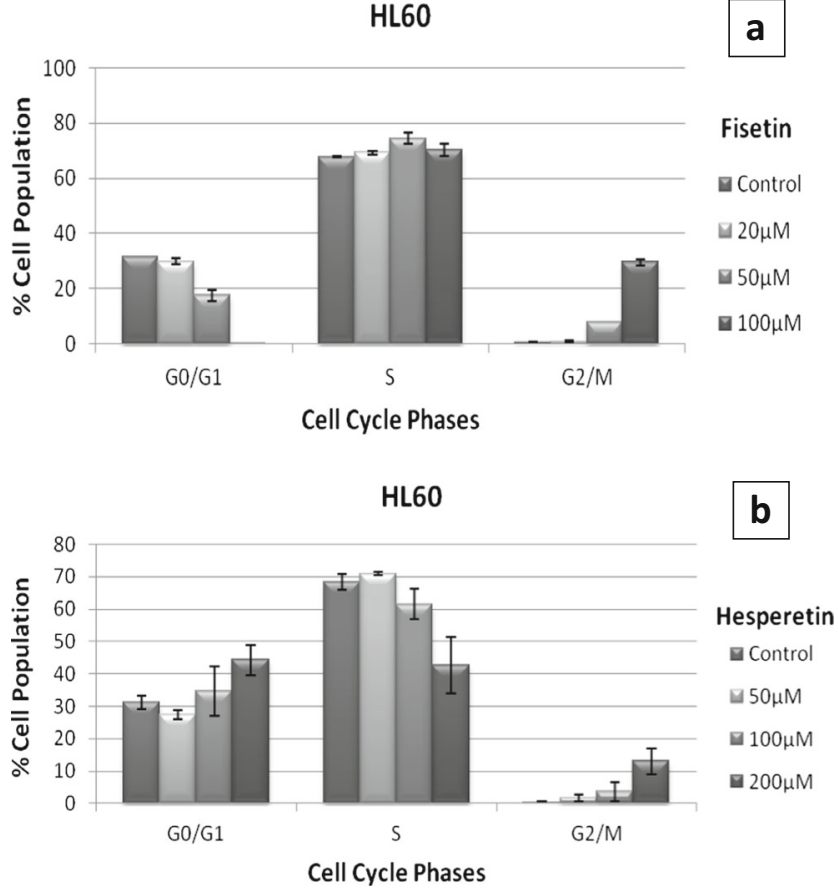

Fig. 5 Effects of fisetin (a) and hesperetin (b) on cell cycle progression of HL60 cells after treatment. The results, the means of three independent experiments, are presented as mean $\pm \mathrm{SEM}$; when not seen, they are smaller than the thickness of the lines on the graphs. Statistical significance was determined using two-way analysis of variance and $P<0.05$ was considered to be significant

\section{Genetic networks affected by fisetin and hesperetin in $\mathbf{H L 6 0}$ cells}

To examine the affected genetic networks after fisetin and hesperetin treatment, we carried out pathway analysis using the IPA tool. These networks describe functional relationships between gene products based on known interactions in the literature. The results showed that the MAPK signaling pathway, ID signaling pathway, cell cycle pathway, JAK/STAT signaling pathway, cell cycle checkpoint pathways, and PI3K/AKT signaling pathways were examples of the most altered networks in fisetin-treated HL60 cells (Table 3).

Based on the data obtained from the IPA analysis, the ID signaling pathway, eukaryotic ribosome and translationrelated networks, gluconeogenesis-related and mitosisrelated networks, and TGF- $\beta$ and MAPK pathways were the affected signaling networks in hesperetin-treated HL60 cells (Table 4).

\section{Discussion}

APL accounts for $10 \%$ of AML. It evolves very rapidly and causes sudden hemorrhages; thus, it is extremely malignant [1]. Currently, the treatment for APL includes chemotherapy, ATRA, ATO, monoclonal antibodies, and hematopoietic stem cell transplantation [2]. Harmful side effects, high recurrence rate and development of resistance to chemotherapeutics and other agents, and heterogeneity in APL cases make APL treatment difficult; thus, they remain incurable in most cases [3]. Thus, new compounds treating APL are urgently needed to be developed.

Flavonoids are known to block the initiation, promotion, and progression of cancer by modulating various signaling pathways that have significant roles in cell proliferation, differentiation, apoptosis, angiogenesis, and metastasis [5]. Thus, flavonoids (both natural and synthetic analogs) are being investigated for their potentials in cancer therapy. Fisetin and hesperetin, plant-derived flavonoids, have been shown to produce a wide range of pharmacological effects including antioxidant, anti-inflammatory, and anticancer effects and found to induce apoptosis in various tumor cells by causing alterations in various cellular processes $[7,13]$. The potential anticarcinogenic effects of fisetin and hesperetin, naturally occurring flavonoids, have been considered in various cancer types especially solid tumors including lung, colon, and breast cancers through several different mechanisms [11, 12, 16, 17]. However, to our knowledge, there is no detailed study investigating their mechanisms of action in APL.

In this study, we measured the time- and dose-dependent effects of fisetin and hesperetin on APL cell viability using the MTT assay. As indicated in the "Results" section, the growth of APL cells was inhibited by fisetin in a dose- and timedependent way while hesperetin was most effective in a longer incubation time. The evaluation of changes in MMP and caspase-3 enzyme activity showed that fisetin and hesperetin treatment results in increases in the loss of MMP and caspase3 enzyme activity with different extents. Induction of apoptosis was also confirmed by localization of phosphatidylserine to the outer membrane after treatment by flow cytometry. We detected increased percentage of cells undergoing apoptosis in a dose-dependent manner for both flavonoids. Therefore, we display that fisetin and hesperetin not only inhibit the proliferation but also induce the apoptosis of APL cells in vitro via mitochondrial membrane depolarization and increased caspase-3 enzyme activity, which suggested that they may have potential to treat APL.

The results of our study are in accordance with the data in the literature derived from various cancer types treated with fisetin. For instance, in LNCaP prostate cancer cells, fisetinmediated apoptosis was related to activation of caspase cascade [19]. In a recent study, Kang et al. [20] displayed that fisetin had a dose-dependent cytotoxic effect on NCI-H460 human non-small cell lung cancer cells, and apoptosis induction in these cells was related to mitochondrial membrane depolarization and caspase-3 activation. Fisetin was also responsible for growth inhibition of U266 multiple myeloma cells via caspase-3 activation [21]. Similarly, hesperetin was displayed to trigger cytotoxicity in MCF-7 breast cancer cells 
Fig. 6 Hierarchical clustering based on gene expression data from HL60 cells exposed to fisetin (upper panel) and hesperetin (lower panel)
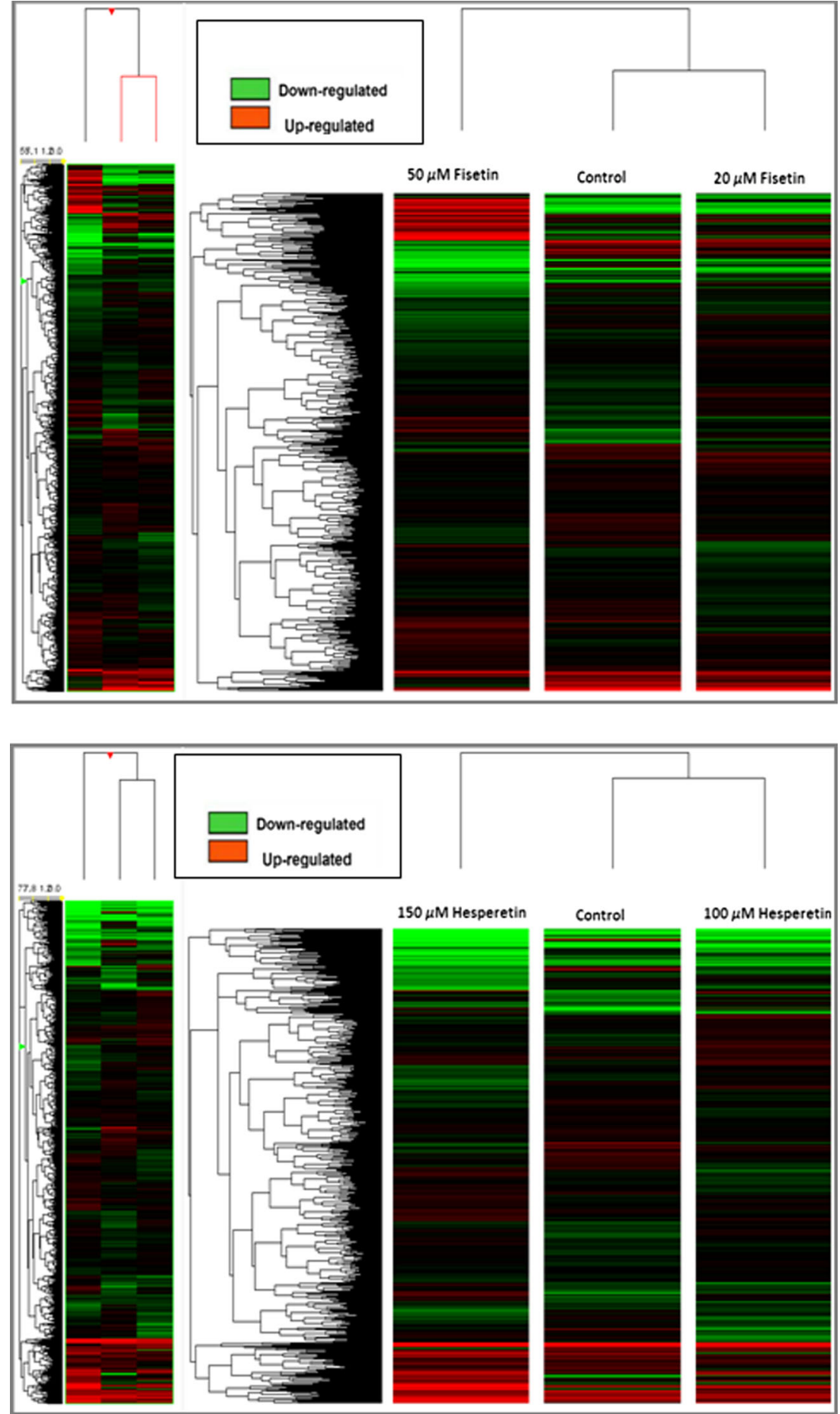

via phosphatidylserine externalization and MMP loss [22]. In another study, SiHa cervical cancer cells treated with hesperetin underwent apoptosis evidenced by disruption of MMP and caspase-3 activation [23].

It is very well known that the cell cycle plays a critical role in cell proliferation, growth, and division [24], and cell cycle arrest could be a reason for growth inhibitory effects of fisetin and hesperetin. Therefore, we evaluated cell cycle distribution of APL cells after fisetin and hesperetin treatment as compared to untreated control cells. Our study shows that fisetin and hesperetin treatment leads to G2/M arrest. The cell cycle progression was also arrested at the G0/G1 phase at 100 and $200 \mu \mathrm{M}$ hesperetin. Previous studies demonstrated that fisetin and hesperetin inhibited the proliferation of cancer cells by inducing G2/M or G0/G1 arrest. For instance, fisetin induced G2/M arrest in human epidermoid carcinoma A431 cells [25], 
Table 1 The list of genes altered $\geq 2$-fold after fisetin treatment of HL60 cells together with their key roles (italic type represents upregulated genes)

\begin{tabular}{|c|c|c|c|}
\hline $\begin{array}{l}\text { Altered genes in fisetin-treated } \\
\text { HL60 cells }\end{array}$ & \multicolumn{2}{|c|}{ Fold change } & Function \\
\hline$T X N I P$ & 11.76 & 12.61 & Apoptosis, tumor suppressor \\
\hline $\operatorname{miRNA1974}$ & 2.496 & 4.23 & Inhibits cell growth \\
\hline TFPI & 2.055 & 3.680 & Inhibitor of invasion and metastasis \\
\hline ID3 & -7.798 & -8.667 & $\begin{array}{l}\text { Dominant negative regulator of basic } \\
\text { helix-loop-helix transcription regulators }\end{array}$ \\
\hline ID1 & -3.685 & -7.005 & $\begin{array}{l}\text { Dominant negative regulator of basic } \\
\text { helix-loop-helix transcription regulators }\end{array}$ \\
\hline HSPA1B & -3.313 & -3.305 & Heath shock response \\
\hline IDH1 & -2.685 & -5.940 & Cytosolic enzyme \\
\hline $\begin{array}{l}\text { Altered genes in } 50-\mu \mathrm{M} \text {-fisetin- } \\
\text { treated HL60 cells }\end{array}$ & \multicolumn{2}{|c|}{ Fold change } & Function \\
\hline$M A P 3 K 1$ & \multicolumn{2}{|l|}{3.310} & Apoptosis, caspase-3 substrate \\
\hline$C B L B$ & \multicolumn{2}{|l|}{3.121} & E3 ubiquitin ligase, autophagy \\
\hline LASS6 & \multicolumn{2}{|l|}{2.728} & Ceramide synthase, apoptosis \\
\hline Caspase-4 & \multicolumn{2}{|l|}{2.422} & Apoptosis \\
\hline LONP1 & \multicolumn{2}{|l|}{-6.017} & Tumor bioenergetic \\
\hline STAT5A & \multicolumn{2}{|l|}{-3.604} & Cell proliferation \\
\hline PHB2 & \multicolumn{2}{|l|}{-3.582} & Cell survival through the Ras pathway \\
\hline XRCC6 & \multicolumn{2}{|l|}{-3.325} & DNA repair \\
\hline PIM2 & \multicolumn{2}{|l|}{-2.960} & Kinase in cell survival \\
\hline PRKCB & \multicolumn{2}{|l|}{-2.920} & Protumorigenic \\
\hline MKNK2 & \multicolumn{2}{|l|}{-2.883} & MAP kinase pathway \\
\hline MAP2K5 & \multicolumn{2}{|l|}{-2.477} & MAP kinase pathway \\
\hline PIK3CB & \multicolumn{2}{|l|}{-2.445} & PI3 kinase pathway, cell proliferation \\
\hline CHECK2 & \multicolumn{2}{|l|}{-2.382} & Cell cycle \\
\hline STAT3 & \multicolumn{2}{|l|}{-2.390} & Cell proliferation \\
\hline GAB2 & \multicolumn{2}{|l|}{-2.168} & PI3 kinase pathway, cell proliferation \\
\hline JAK1 & \multicolumn{2}{|l|}{-2.045} & Cell proliferation \\
\hline
\end{tabular}

HT-29 colon cancer cells [8], and PC3 prostate cancer cells [26]. Human cervical SiHa cells treated with hesperetin displayed G2/M arrest in a dose-dependent manner as well [23]. Fisetin and hesperetin blocked cell cycle progression at G0/G1 in T24 and E7 bladder and MCF-7 breast cancer cells by decreasing cyclin-dependent kinases and cyclins involved in the regulation of G0/G1 passage [27, 17]. Therefore, our data together with literature knowledge explain that these two flavonoids may arrest cell cycle in a cell-type-specific manner and, thus, act as a non-specific cell cycle blocker.

We also performed genome-wide gene profiling analysis to establish genes affected in response to fisetin and hesperetin. Tables 1 and 2 summarize differentially expressed genes constrained by $P$ value $<0.05$ and at least 2.0 fold change. As clearly seen in Table 1, TXNIP, miRNA1974, and TFPI genes were common upregulated genes, which are known to be involved in the inhibition of cell growth, invasion, and metastasis. For instance, TXNIP or thioredoxin-interacting protein plays a crucial role in apoptosis. Its expression was found to be downregulated in many tumor cells [28], and overexpression of TXNIP inhibits cancer cell growth [29].
Another upregulated gene, TFPI, an inhibitor of plasminactivating matrix metalloproteinases, blocks invasion and metastasis [30]. Downregulation of some common genes such as ID1, ID3, and IDH1 in fisetin-treated HL60 cells could be responsible for its apoptotic effects as well. In a recent study, suppression of ID1 and ID3, transcription regulators involved in proliferation and apoptosis, was related to reduced tumor growth [31, 32]. Downregulation of IDH1 after fisetin treatment might cause cell death due to the accumulation of reactive oxygen species (ROS) that damage proteins, lipids, and DNA in the cells, since it is involved in the production of NADPH required to scavenge ROS [33]. It is also understood from Table 1 that additional genes encoding proteins with significant functions in important cancer-related signaling pathways such as the MAPK pathway (MKNK2, MAP2K5), PI3K pathway (PIK3CB, GAB2), and JAK/STAT pathway (STAT5A, STAT3, and JAK1) were downregulated in response to higher fisetin concentration, thus leading to suppression of leukemic cell growth and induction of apoptosis. LONP1 gene expression was significantly downregulated at higher fisetin concentration, and it could be also an important 
Table 2 The list of genes altered $\geq 2$-fold after hesperetin treatment of HL60 cells including their functions in the cell (italic type represents upregulated genes)

\begin{tabular}{|c|c|c|c|}
\hline $\begin{array}{l}\text { Altered genes in hesperetin-treated } \\
\text { HL60 cells }\end{array}$ & \multicolumn{2}{|c|}{ Fold change } & Function \\
\hline$S P R R 2 D$ & 4.574 & 5.985 & Differentiation \\
\hline$M T 1 F$ & 2.758 & 4.851 & Tumor suppressor \\
\hline SASH1 & 2.508 & 3.023 & Tumor suppressor \\
\hline S100P & -8.285 & -10.153 & Cell proliferation \\
\hline ID1 & -5.467 & -6.361 & $\begin{array}{l}\text { Dominant negative regulator of basic } \\
\text { helix-loop-helix transcription regulators }\end{array}$ \\
\hline NMU & -5.062 & -6.065 & Invasion, metastasis \\
\hline ID3 & -3.913 & -4.256 & $\begin{array}{l}\text { Dominant negative regulator of basic } \\
\text { helix-loop-helix transcription regulators }\end{array}$ \\
\hline FGFR3 & -3.270 & -3.623 & Cell proliferation \\
\hline TUBB1 & -3.010 & -3.327 & Cell division, tubulin isotype \\
\hline $\begin{array}{l}\text { Altered genes in } 150-\mu \mathrm{M}- \\
\text { hesperetin-treated HL60 cells }\end{array}$ & \multicolumn{2}{|c|}{ Fold change } & Function \\
\hline$T X N I P$ & \multicolumn{2}{|l|}{2.977} & Apoptosis, tumor suppressor \\
\hline$M T 1 G$ & \multicolumn{2}{|l|}{2.939} & Tumor suppressor \\
\hline TPM1 & \multicolumn{2}{|l|}{2.734} & Tropomyosin 1, tumor suppressor \\
\hline$S P R R 2 F$ & \multicolumn{2}{|l|}{2.673} & Differentiation \\
\hline$R E R G$ & \multicolumn{2}{|l|}{2.629} & Ras-related growth inhibitor \\
\hline ST7 & \multicolumn{2}{|l|}{2.609} & Tumor suppressor, cell cycle arrest \\
\hline$M A P 3 K 1$ & \multicolumn{2}{|l|}{2.606} & Apoptosis, caspase-3 substrate \\
\hline RASAl & \multicolumn{2}{|l|}{2.438} & Inhibitor of mitogenic signal \\
\hline MT1A & \multicolumn{2}{|l|}{2.434} & Tumor suppressor \\
\hline $\operatorname{SIN} 3 A$ & \multicolumn{2}{|l|}{2.400} & Tumor suppressor \\
\hline RPS25 & \multicolumn{2}{|l|}{-8.177} & Ribosome component \\
\hline TUBA1C & \multicolumn{2}{|l|}{-3.544} & Cell division, tubulin isotype \\
\hline EEF1A1 & \multicolumn{2}{|l|}{-3.042} & Protein translation \\
\hline RPS6P1 & \multicolumn{2}{|l|}{-3.029} & Ribosome component \\
\hline PRKCB1 & \multicolumn{2}{|l|}{-2.432} & Cell proliferation \\
\hline C-MYC & \multicolumn{2}{|l|}{-2.157} & Proto-oncogene transcription factor \\
\hline PIM1 & \multicolumn{2}{|l|}{-2.330} & Proto-oncogene kinase \\
\hline TUBB2C & \multicolumn{2}{|l|}{-2.226} & Cell division \\
\hline RPS9 & \multicolumn{2}{|l|}{-2.208} & Ribosome component \\
\hline PCK2 & \multicolumn{2}{|l|}{-2.206} & Enzyme involved in gluconeogenesis \\
\hline RXRA & \multicolumn{2}{|l|}{-2.122} & Differentiation \\
\hline
\end{tabular}

Table 3 Affected networks in fisetin-treated HL60 cells by IPA analysis (italic type represents networks modulated in both 20- and 50$\mu \mathrm{M}$ fisetin treatment)

\begin{tabular}{ll}
\hline Network/pathway & $P$ value \\
\hline MAPK signaling pathway $(50 \mu \mathrm{M})$ & 0.04 \\
ID signaling pathway & $2.71 \mathrm{E}-05(20 \mu \mathrm{M})$ \\
& $3.62 \mathrm{E}-04(50 \mu \mathrm{M})$ \\
Nucleotide excision repair $(50 \mu \mathrm{M})$ & $1.48 \mathrm{E}-06$ \\
Cell cycle pathway $(50 \mu \mathrm{M})$ & $2.47 \mathrm{E}-05$ \\
JAK/STAT signaling pathway $(50 \mu \mathrm{M})$ & 0.002 \\
Protein folding & $0.01(20 \mu \mathrm{M})$ \\
& $2.01 \mathrm{E}-09(50 \mu \mathrm{M})$ \\
Cell cycle checkpoint pathways $(50 \mu \mathrm{M})$ & $2.57 \mathrm{E}-04$ \\
PI3K/AKT pathway $(50 \mu \mathrm{M})$ & 0.04 \\
\hline
\end{tabular}

Table 4 Affected networks in hesperetin-treated HL60 cells by IPA analysis (italic type represents networks modulated in both 100- and $150-\mu \mathrm{M}$ hesperetin treatment)

\begin{tabular}{ll}
\hline Network/pathway & $P$ value \\
\hline ID signaling pathway & $4.52 \mathrm{E}-04(100 \mu \mathrm{M})$ \\
& $0.008(150 \mu \mathrm{M})$ \\
TGF- $\beta$ pathway & $7.45 \mathrm{E}-04(100 \mu \mathrm{M})$ \\
& $3.32 \mathrm{E}-04(150 \mu \mathrm{M})$ \\
Cytoplasmic ribosomal components $(150 \mu \mathrm{M})$ & $1.27 \mathrm{E}-10$ \\
Translation factors $(150 \mu \mathrm{M})$ & $4.24 \mathrm{E}-06$ \\
Gluconeogenesis $(150 \mu \mathrm{M})$ & $4.29 \mathrm{E}-04$ \\
Mitosis $(150 \mu \mathrm{M})$ & 0.001 \\
MAPK signaling pathway $(150 \mu \mathrm{M})$ & 0.01 \\
\hline
\end{tabular}


target to induce leukemic cell death because this gene is found to be overexpressed in different tumors and cell lines [34].

The genome-wide changes in the expression levels of genes after hesperetin treatment in HL60 APL cells are summarized in Table 2. SPRR2D, MT1F, and SASH1 were upregulated genes that are known to play roles in cell proliferation, apoptosis, and differentiation. It is known that cancer cells have no or lower expression of SPRRs compared to normal cells [35]. Therefore, increased expression of SPRR2D is thought to induce cell growth suppression with an unknown mechanism. MT1F, a member of the metallothionein family, is expressed transiently in response to stimulation and plays an important role in cell growth by modulating gene transcription [36]. In a recent study, exogenous overexpression of the MT1F gene increased apoptosis in colon cancer cells [37], which supports our data. SASH1, a potential tumor suppressor, may function in various signaling pathways due to its SH3 and SAM domains; thus, its downregulation is related to tumor growth [38]. Therefore, its increased expression after hesperetin treatment might induce apoptosis and growth suppression. Similar to fisetin treatment as discussed above, ID1 and ID3 genes were downregulated in hesperetin treatment, thus inducing apoptosis. The other common downregulated gene was NMU, which was found to be increased in AML cells, and its knockdown caused growth arrest [39]. Therefore, it may be a reason for hesperetin-related antiproliferative effect. Another significantly downregulated gene was S100P, whose upregulation results in increased cell proliferation in several tumors such as endometrial cancer and oral squamous cell carcinoma [40, 41]. As well as these common altered genes, higher hesperetin treatment also induced either up- or downregulation of several genes as indicated in Table 2. It is interpreted from Table 2 that increased hesperetin concentration resulted in downregulation of additional tubulin-encoding genes, TUBB2C and TUBA1A, which could cause problems in cell division and then induce apoptosis [42]. Hesperetin impaired protein synthesis by targeting cytoplasmic ribosomal proteins such as RPS6P1, RPS9, and RPS25 and factors involved in translation initiation like EEF1A1. Another important downregulated gene was C-MYC which is described as a proto-oncogenic transcription factor and upregulated in various cancer types such as leukemia, lung, and breast cancers, thus inducing cell proliferation and inhibition of apoptosis via altering the expression of several target genes [43]. Therefore, its downregulation by hesperetin could be a strategy to inhibit HL60 cell growth. PIM1, a member of serine/threonine kinases involved in cell survival and proliferation, is overexpressed in a variety of cancer types such as leukemias, lymphomas, and pancreatic and prostate cancers [44]. It is also known that PIM1 functions together with C-MYC to induce malignancy. In our study, hesperetin downregulates both $\mathrm{C}$ MYC and PIM1, therefore inducing significant growth inhibition and apoptosis.
We also characterize differentially modulated networks in response to fisetin and hesperetin using the IPA tool. Genes associated with biological functions are taken into consideration for analysis to identify networks, showing physical or functional relationships among differentially expressed genes. As clearly seen in Table 3, IPA analysis of fisetin-induced pathways includes ID signaling, JAK/STAT, and MAPK kinase signaling pathways. On the other hand, Table 4 displays networks/pathways modulated after hesperetin treatment, which include networks involved in protein synthesis (cytoplasmic ribosomal components, translation factors) and cell division. All these data are consistent with gene expression profiles.

\section{Conclusions}

The present study has revealed that fisetin and hesperetin have significant antiproliferative effects on HL60 APL cells evidenced by induction of apoptosis and cell cycle arrest. Based on the data presented, fisetin and hesperetin trigger apoptosis and growth suppression via affecting various significant targets as in HL60 cells, and these new targets could open the usage of new strategies together with fisetin and hesperetin to overcome difficulties in APL treatment. These data also provide significant insights about the mechanisms of action of fisetin and hesperetin in HL60 cells for the first time. Further in vivo investigations are needed to confirm its antitumor activity and toxicity profiles.

Acknowledgements Declared none

Conflicts of interest None

\section{References}

1. Lo-Coco F, Hasan SK. Understanding the molecular pathogenesis of acute promyelocytic leukemia. Best Pract Res Clin Haematol. 2014;27:3-9.

2. de Thé H, Le Bras M, Lallemand-Breitenbach V. Acute promyelocytic leukemia, arsenic, and PML bodies. J Cell Biol. 2012;198:11-21.

3. Mi J. Current treatment strategy of acute promyelocytic leukemia. Front Med. 2011;5:341-7.

4. Khan N, Adhami VM, Mukhtar H. Apoptosis by dietary agents for prevention and treatment of cancer. Biochem Pharmacol. 2008;76: 1333-9.

5. Ravishankar D, Rajora AK, Greco F, Osborn HM. Flavonoids as prospective compounds for anti-cancer therapy. Int J Biochem Cell Biol. 2013;45:2821-31.

6. Khan N, Syed DN, Ahmad N, Mukhtar H. Fisetin: a dietary antioxidant for health promotion. Antioxid Redox Signal. 2013;19: 151-62.

7. Syed DN, Suh Y, Afaq F, Mukhtar H. Dietary agents for chemoprevention of prostate cancer. Cancer Lett. 2008;265:167-76. 
8. Lu X, Jung J, Cho HJ, Lim DY, Lee HS, Chun HS, et al. Fisetin Inhibits the activities of cyclin-dependent kinases leading to cell cycle arrest in HT-29 human colon cancer cells. J Nutr. 2005;135:122884-90.

9. Murtaza I, Adhami VM, Hafeez BB, Saleem M, Mukhtar H. Fisetin, a natural flavonoid, targets chemoresistant human pancreatic cancer AsPC-1 cells through DR3 mediated inhibition of NFKB. Int J Cancer. 2009;2:2465-73.

10. Adhami VM, Syed DN, Khan N, Mukhtar H. Dietary flavonoid fisetin: a novel dual inhibitor of PI3K/Akt and mTOR for prostate cancer management. Biochem Pharmacol. 2012;84:1277-81.

11. Liao YC, Shih YW, Chao CH, Lee XY, Chiang TA. Involvement of the ERK signaling pathway in fisetin reduces invasion and migration in the human lung cancer cell line A549. J Agric Food Chem. 2009;57:8933-41.

12. Lim do Y, Park JH. Induction of p53 contributes to apoptosis of HCT116 human colon cancer cells induced by the dietary compound fisetin. Am J Physiol Gastrointest Liver Physiol. 2009;296(5): G1060-8.

13. Benavente-Garcia O, Castillo J. Update on uses and properties of citrus flavonoids: new findings in anticancer, cardiovascular, and anti-inflammatory activity. J Agric Food Chem. 2008;56:6185-205.

14. Nielsen IL, Chee WS, Poulsen L, Offord-Cavin E, Rasmussen SE, et al. Frederiksen, bioavailability is improved by enzymatic modification of the citrus flavonoid hesperidin in humans: a randomized, double-blind, crossover trial. J Nutr. 2006;136:404-8.

15. Chen YC, Shen SC, Lin HY. Rutinoside at C7 attenuates the apoptosis-inducing activity of flavonoids. Biochem Pharmacol. 2003;66:1139-50.

16. Sivagami G, Vinothkumar R, Bernini R, Preethy CP, Riyasdeen A, Akbarsha MA, et al. Role of hesperetin (a natural flavonoid) and its analogue on apoptosis in HT-29 human colon adenocarcinoma cell line - a comparative study. Food Chem Toxicol. 2012;50:660-71.

17. Choi EJ. Hesperetin induced G1-phase cell cycle arrest in human breast cancer MCF-7 cells: involvement of CDK4 and p21. Nutr Cancer. 2007;59:115-9.

18. Green DR, Reed JC. Mitochondria and apoptosis. Science. 1998;281:1309-12.

19. Khan N, Afaq F, Syed DN, Mukhtar H. Fisetin, a novel dietary flavonoid, causes apoptosis and cell cycle arrest in human prostate cancer LNCaP cells. Carcinogenesis. 2008;29:1049-56.

20. Kang KA, Piao MJ, Hyun JW. Fisetin induces apoptosis in human nonsmall lung cancer cells via a mitochondria-mediated pathway. In Vitro Cell Dev Biol Anim. 2015;51:300-9.

21. Jang KY, Jeong SJ, Kim SH, Jung JH, Kim JH, Koh W, et al. Activation of reactive oxygen species/AMP activated protein kinase signaling mediates fisetin induced apoptosis in multiple myeloma U266 cells. Cancer Lett. 2012;319:197-202.

22. Palit S, Kar S, Sharma G, Das PK. Hesperetin induces apoptosis in breast carcinoma by triggering accumulation of ROS and activation of ASK1/JNK pathway. J Cell Physiol. 2015;230(8):1729-39.

23. Alshatwi AA, Ramesh E, Periasamy VS, Subash-Babu P. The apoptotic effect of hesperetin on human cervical cancer cells is mediated through cell cycle arrest, death receptor, and mitochondrial pathways. Fundam Clin Pharmacol. 2013;27:581-92.

24. Peyressatre M, Prével C, Pellerano M, Morris MC. Targeting cyclin-dependent kinases in human cancers: from small molecules to peptide inhibitors. Cancers (Basel). 2015;7(1):179-237.

25. Pal HC, Sharma S, Elmets CA, Athar M, Afaq F. Fisetin inhibits growth, induces $\mathrm{G} 2 / \mathrm{M}$ arrest and apoptosis of human epidermoid carcinoma A431 cells: role of mitochondrial membrane potential disruption and consequent caspases activation. Exp Dermatol. 2013;22:470-5.

26. Haddad AQ, Venkateswaran V, Viswanathan L, Teahan SJ, Fleshner NE, Klotz LH. Novel antiproliferative flavonoids induce cell cycle arrest in human prostate cancer cell lines. Prostate Cancer Prostatic Dis. 2006;9:68-76.
27. Li J, Cheng Y, Qu W, Sun Y, Wang Z, Wang H, et al. Fisetin, a dietary flavonoid, induces cell cycle arrest and apoptosis through activation of $\mathrm{p} 53$ and inhibition of NF-kappa B pathways in bladder cancer cells. Basic Clin Pharmacol Toxicol. 2011;108:84-93.

28. Escrich E, Moral R, Garcia G, Costa I, Sanchez JA, Solanas M. Identification of novel differentially expressed genes by the effect of a high-fat $\mathrm{n}-6$ diet in experimental breast cancer. Mol Carcinog. 2004;40:73-8.

29. Yamaguchi F, Hirata Y, Akram H, Kamitori K, Dong Y, Sui L, et al. FOXO/TXNIP pathway is involved in the suppression of hepatocellular carcinoma growth by glutamate antagonist MK-801. BMC Cancer. 2013;13:468. doi:10.1186/1471-2407-13-468.

30. Lavergne M, Jourdan ML, Blechet C, Guyetant S, Pape AL, HeuzeVourc'h N, et al. Beneficial role of overexpression of TFPI2 on tumour progression in human small cell lung cancer. FEBS Open Bio. 2013;3:291-301.

31. Norton JD. ID helix-loop-helix proteins in cell growth, differentiation and tumorigenesis. J Cell Sci. 2000;113:3897-905.

32. Chen D, Forootan SS, Gosney JR, Forootan FS, Ke Y. Increased expression of ID1 and ID3 promotes tumorigenicity by enhancing angiogenesis and suppressing apoptosis in small cell lung cancer. Genes Cancer. 2014;5:212-25.

33. Kim S, Kim SY, Ku HJ, Jeon YH, Lee HW, Lee J, et al. Suppression of tumorigenesis in mitochondrial $\mathrm{NADP}(+)$-dependent isocitrate dehydrogenase knock-out mice. Biochim Biophys Acta. 1842;2014:135-43.

34. Bota DA, Ngo JK, Davies KJ. Downregulation of the human Lon protease impairs mitochondrial structure and function and causes cell death. Free Radic Biol Med. 2005;38:665-77.

35. Anisowicz A, Sotiropoulou G, Sager R. Re-expression of SPR1 in breast cancer cells by phorbol 12-myristate 13-acetate (PMA) or UV irradiation is mediated by the AP-1 binding site in the SPR 1 promoter. Mol Med. 1999;5:526-41.

36. Lu DD, Chen YC, Zhang XR, Cao XR, Jiang HY, Yao L. The relationship between metallothionein-1F (MT1F) gene and hepatocellular carcinoma. Yale J Biol Med. 2003;76:55-62.

37. Yan DW, Fan JW, Yu ZH, Li MX, Wen YG, Li DW, et al. Downregulation of metallothionein $1 \mathrm{~F}$, a putative oncosuppressor, by loss of heterozygosity in colon cancer tissue. Biochim Biophys Acta. 2012;1822(6):918-26.

38. Meng Q, Zheng M, Liu H, Song C, Zhang W, Yan J, et al. SASH1 regulates proliferation, apoptosis, and invasion of osteosarcoma cell. Mol Cell Biochem. 2013;373:201-10.

39. Shetzline SE, Rallapalli R, Dowd KJ, Zou S, Nakata Y, Swider CR, et al. Neuromedin U: a Myb regulated autocrine growth factor for human myeloid leukemias. Blood. 2004;104:1833-40.

40. Guo L, Chen S, Jiang H, Huang J, Jin W, Yao S. The expression of $\mathrm{S} 100 \mathrm{P}$ increases and promotes cellular proliferation by increasing nuclear translocation of $\beta$-catenin in endometrial cancer. Int J Clin Exp Pathol. 2014;7(5):2102-12.

41. Wu TS, Tan CT, Chang CC, Lin BR, Lai WT, Chen ST, et al. B-cell lymphoma/leukemia 10 promotes oral cancer progression through STAT1/ATF4/S100P signaling pathway. Oncogene. 2015;34(10): 1207-19.

42. Leandro-García LJ, Leskelä S, Landa I, Montero-Conde C, LópezJiménez E, Letón R, et al. Tumoral and tissue-specific expression of the major human beta-tubulin isotypes. Cytoskeleton (Hoboken). 2010;67:214-23.

43. Dang CV. c-Myc target genes involved in cell growth, apoptosis, and metabolism. Mol Cell Biol. 1999;19:1-11.

44. Saurabh K, Scherzer MT, Shah PP, Mims AS, Lockwood WW, Kraft AS, et al. The PIM family of oncoproteins: small kinases with huge implications in myeloid leukemogenesis and as therapeutic targets. Oncotarget. 2014;5:8503-14. 